\title{
Computer simulations of the Rescorla-Wagner and Pearce-Hall models in conditioning and contingency judgment
}

\author{
PIERRE MERCIER \\ University of Ottawa, Ottawa, Ontario, Canada
}

\begin{abstract}
A Visual BASIC program, running under Windows 3.1, simulated the predictions of the RescorlaWagner (Rescorla \& Wagner, 1972) and the Pearce-Hall (Pearce \& Hall, 1980) models and compared them to the normative contingency coefficient $\Delta \mathrm{P}$ (Jenkins \& Ward, 1965). The simulations can be applied to a variety of phenomena in human contingency judgment as well as learning and conditioning. Possible simulations include acquisition and extinction of excitatory and inhibitory conditioning, latent inhibition, blocking and overshadowing, or any other associative learning involving two single predictors, their compound, a contextual stimulus, and an outcome. The Pearce-Hall model has never been computerized before. In addition, unique features of this software include extensive use of the graphic user interface, context-sensitive help, verification of trial combinations, toggling of the contextual stimulus from ever-present to mutually exclusive with the discrete predictors, data entry via contingency tables or specifications trial by trial, single or batch randomizations of trial order, and specification of initial values. The associative simulator is both a powerful scientific instrument and a user-friendly teaching aid.
\end{abstract}

Recent research in learning and cognitive psychology (Chapman, 1991; Dickinson, Shanks, \& Evenden, 1984; Gluck \& Bower, 1988; Shanks, 1985a, 1991) draws a parallel between the type of associative learning traditionally studied in conditioning paradigms with animals and the cognitive processes involved in the detection of covariations, the judgment of contingency and causality, and categorization. Because of this, there is renewed interest in formal models of associative learning, chiefly among them, the Rescorla-Wagner (Rescorla \& Wagner, 1972) model.

Two simulators of the Rescorla-Wagner model have been published before. One was designed mainly for teaching purposes and ran on Commodore 64 computers (Bond \& Siddle, 1987). It also had the capability to simulate Solomon and Corbit's (1974) opponent process theory. Interest in the opponent process theory has decreased over the years because it did not achieve the same range of powerful predictions as did the Rescorla-Wagner model, primarily with regard to stimulus competition. Additionally, C-64 computers have been superseded by IBM compatibles and Macintoshes as the main laboratory computer equipment.

The other program (Lachnit, Schneider, Lipp, \& Kimmel, 1988) is menu-driven and can compute associative strengths for differential conditioning, conditioned inhibition, compound and contextual conditionings, transswitching, and other designs. Although versatile, this pro-

Correspondence should be addressed to P. Mercier, School of Psychology, University of Ottawa, Ottawa, ON, Canada, KIN 6N5 (e-mail: nitch@locutus.cc.uottawa.ca). gram does not simulate the Pearce-Hall (Pearce \& Hall, 1980) model and lacks a number of useful features (described below).

\section{THE RESCORLA-WAGNER MODEL}

The Rescorla-Wagner model was designed to account for the variations in associative strength resulting from pairing or unpairing conditional stimuli and reinforcers. More importantly, it was also designed to account for the existence of selective associations in learning. Selective associations occur when the associative strength accruing to a stimulus is stronger or weaker than what would be anticipated strictly on the basis of the contiguous reinforcement history of that stimulus. One such selective association is called blocking.

Blocking occurs when a conditioned Stimulus B is reinforced from its first presentation in compound with another Stimulus A, which is already strongly conditioned. In this case, $\mathrm{B}$ becomes less conditioned than it otherwise would if compounded with a neutral A. Originally reported in studies of Pavlovian conditioning (Kamin, 1969), the phenomenon has been replicated numerous times (Dickinson, Hall, \& Mackintosh, 1976; Mackintosh, 1971, 1973, 1975, 1978; Mackintosh, Bygrave, \& Picton, 1977; Mackintosh \& Turner, 1971; Turner \& Mackintosh, 1972) in the same paradigm and has also been reported in more recent studies of human contingency judgment (Chapman, 1991; Chapman \& Robbins, 1990; Shanks, 1985b, 1986, 1989).

The Rescorla-Wagner model computes variations in associative strength with a set of three fundamental equations. The first equation defines the summation of associative strength for all stimuli present on a given trial: 


$$
V_{\Sigma}^{n}=V_{A}^{n}+\ldots V_{X}^{n} .
$$

The second equation determines the amount of change for a given stimulus on a given trial:

$$
\Delta V_{A}^{n}=\alpha_{A} \beta_{1}\left(\lambda-V_{\Sigma}^{n-1}\right) .
$$

The expression indicates that the change in the associative strength of Stimulus A on Trial $n\left(\Delta V_{A}^{n}\right)$ is a function of the salience of that stimulus $\left(\alpha_{A}\right)$ times the salience of the reinforcer $\left(\beta_{1}\right)$ times the difference between the maximum strength possible $(\lambda)$ and the previously accumulated strength to all stimuli present on that trial $\left(V_{\Sigma}^{n-1}\right)$.

A final equation cumulates associative strength over trials:

$$
V_{A}^{n}=V_{A}^{n-1}+\Delta V_{A}^{n}
$$

In the simple case of one continuously reinforced conditioned stimulus, $\mathrm{A}$, the equations would generate a negatively accelerated growth function.

In the case of blocking, two stimuli become conditioned. Reinforcement for A begins first. Following this, A and B are then reinforced in compound. Although $B$ is reinforced many times, the pairings result in less associative strength accruing to $\mathrm{B}$ than if $\mathrm{A}$ had not been preconditioned. A has blocked B by appropriating most of the available associative strength during the early trials (Figure 1).

\section{THE PEARCE-HALL MODEL}

A number of recent studies of human contingency judgment and categorization (Chapman \& Robbins, 1990; Shanks, 1991, 1993) strongly suggest not only that associative models provide good accounts of cue competition in these tasks but also that it would be worthwhile to contrast variants of this type of model. One associative variant, proposed by Pearce and Hall (1980), was directly compared to Rescorla-Wagner by Dickinson et al. (1984).

The Pearce-Hall model was designed in part to accommodate some subtle aspects of blocking as well as additional phenomena such as conditional stimulus (CS) preexposure or "latent inhibition" (Hall \& Pearce, 1982; Lubow \& Moore, 1959; Mercier \& Baker, 1985; Pearce, Nicholas, \& Dickinson, 1982). It differs from Rescorla-Wagner in two important respects: (1) It assumes that predictive stimuli vary in associability rather than reinforcement varying in effectiveness, and (2) it proposes a separate process for the accumulation of negative associative strength.

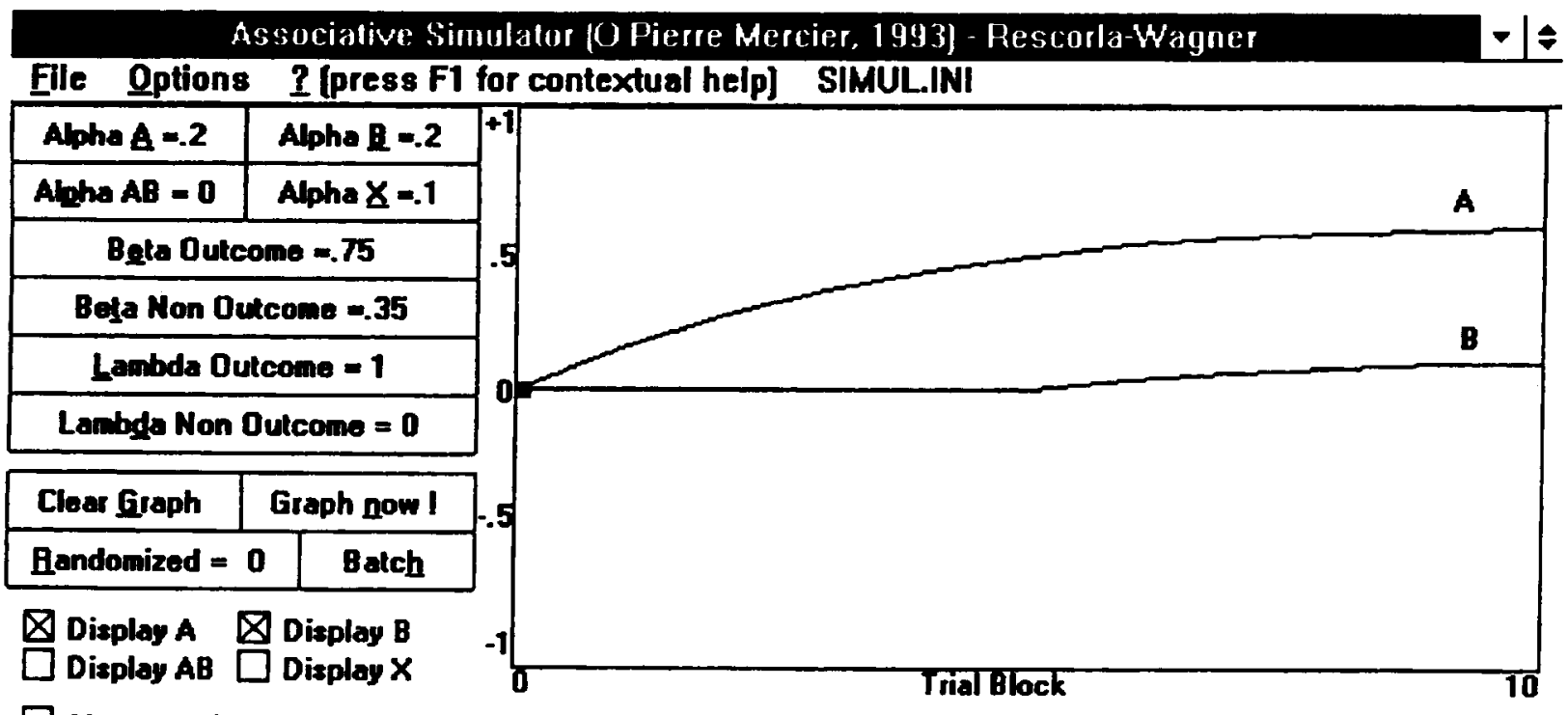

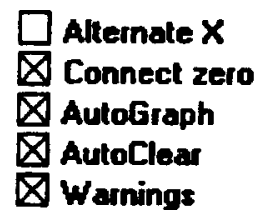

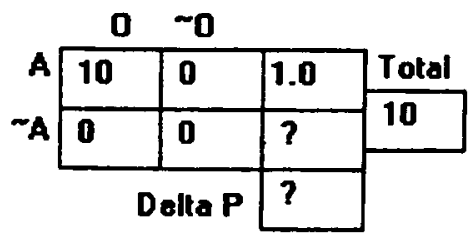

\begin{tabular}{|c|c|c|c|c|c|c|}
\hline Trial & $\mathbf{A}$ & 8 & AB & $x$ & Dutcome & 4 \\
\hline 1 & 1 & 0 & 0 & 1 & 1 & \\
\hline 2 & 1 & 0 & 0 & 1 & 1 & \\
\hline 3 & 1 & 0 & 0 & 1 & 1 & \\
\hline 4 & 1 & $\mathbf{0}$ & 0 & 1 & 1 & \\
\hline 5 & 1 & 0 & 0 & 1 & 1 & \\
\hline 6 & 1 & 1 & 1 & 1 & 1 & \\
\hline 7 & 1 & 1 & 1 & 1 & 1 & \\
\hline 8 & 1 & 1 & 1 & 1 & 1 & \\
\hline 9 & 1 & 1 & 1 & 1 & 1 & \\
\hline 10 & 1 & 1 & 1 & 1 & 1 & $t$ \\
\hline
\end{tabular}
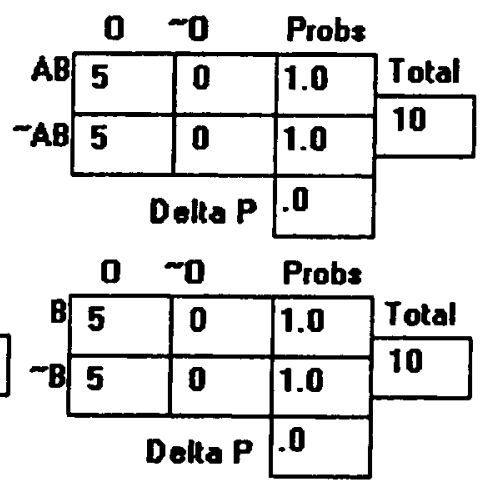

Figure 1. Simulation of blocking according to Rescorla and Wagner. 
The first equation describes how the associability of a stimulus changes across trials:

$$
\Delta v_{A}^{n}=S_{A} \alpha_{A} \lambda^{n},
$$

where $S_{A}$ is the stimulus salience, $\alpha_{A}$ is the stimulus associability, and $\lambda^{n}$ is the maximum associative strength that can be reached on a given trial. The associability of the stimulus is determined by whether or not that stimulus fully predicts its consequences. This is expressed as

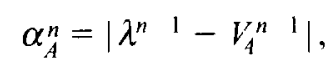

although the complete form of this equation is in Equation 10 (below).

By substituting Equation 5 into 4, we obtain

$$
\Delta V_{A}^{n}=S_{A}\left|\lambda^{n}{ }^{1}-V_{A}^{n-1}\right| \lambda^{n} .
$$

These trial-by-trial changes are accumulated by summing with the existing associative strength using

$$
V_{A}^{n}=V_{A}^{n-1}+\Delta V_{A}^{n}
$$

Negative associative strength accumulates separately and competes with excitation on every trial. Thus, inhibitory strength changes as

$$
\Delta \bar{V}_{A}=S_{A} \alpha_{A} \bar{\lambda}
$$

where

$$
\bar{\lambda}=\left(V_{\Sigma}-\bar{V}_{\Sigma}\right)-\lambda
$$

and $V_{\Sigma}$ and $\bar{V}_{\Sigma}$ are the total excitatory and inhibitory strength accruing to all stimuli present on the trial. Hence, stimulus associability is really determined by

$$
\alpha^{n}=\left|\lambda^{n-1}-\left(V_{\Sigma}^{n} 1-\bar{V}_{\Sigma}^{n-1}\right)\right| .
$$

Applying this model to the blocking example above, we obtain a pattern of associative strength similar to that of Rescorla-Wagner except that while B is blocked, A still continues to grow toward asymptote.

The two models are also different in their ability to predict the retardation in acquisition normally brought about by CS preexposure. The Rescorla-Wagner model cannot really account for this phenomenon unless a mechanism is provided to change stimulus salience across trials. This is exactly the type of mechanism embodied in the PearceHall model, the results of which are shown in Figure 2. In this figure, $A$ is first presented alone for a few trials before reinforcement begins. The simulation for Rescorla-Wagner shows the acquisition of associative strength for $\mathrm{A}$ to be

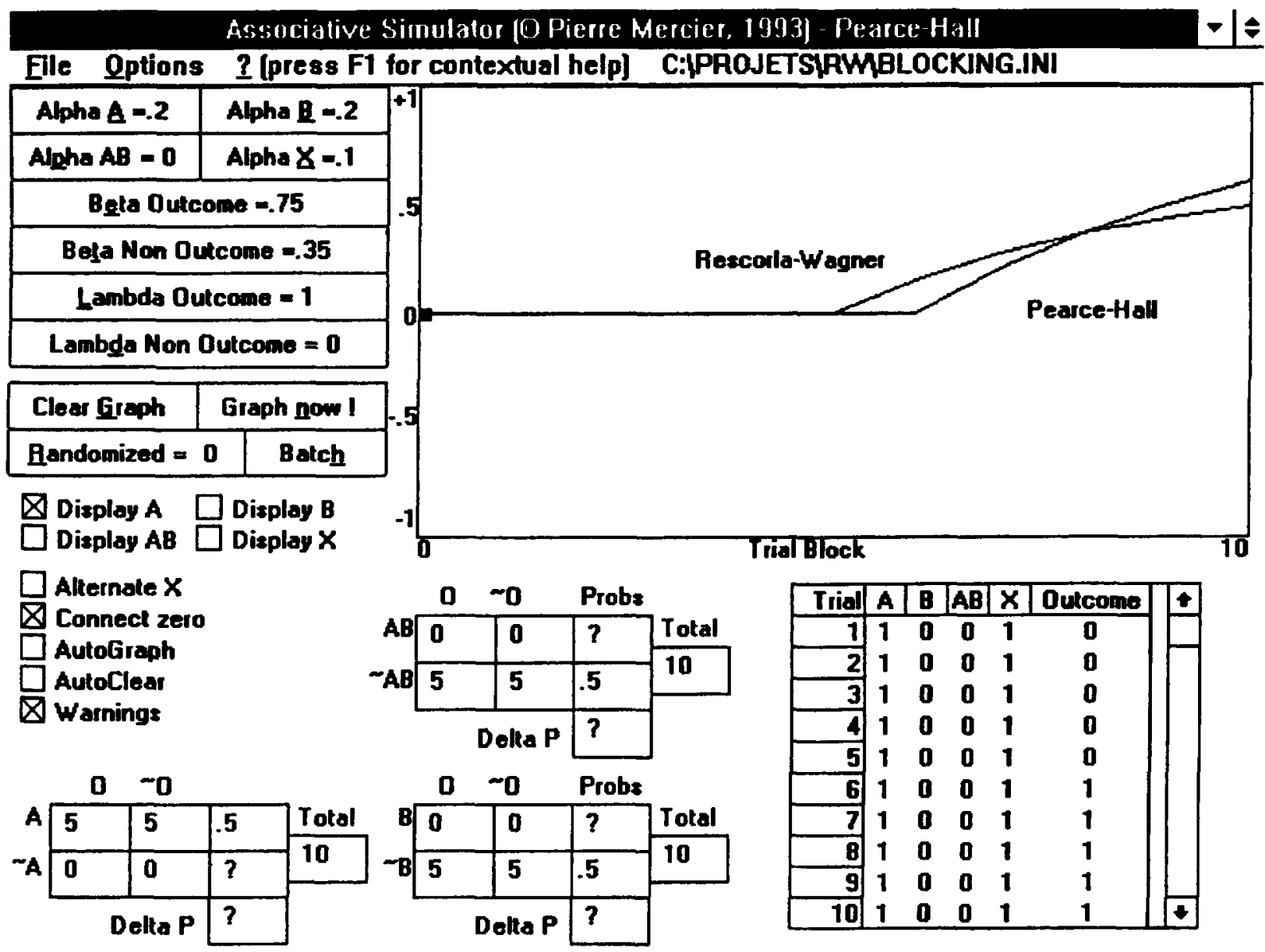

Figure 2. Simulations of conditional stimulus (CS) preexposure (latent inhibition) using the Rescorla-Wagner and the Pearce-Hall models. 
the same as if reinforcement had started on the first trial. The simulation for Pearce-Hall shows a temporary retardation that quickly disappears as reinforced trials succeed.

There are many other differences between the two models, most notably in situations involving inhibitory associations, but the examples should suffice to introduce the simulator.

\section{CONTINGENCY JUDGMENT}

The recent applications of associative models to human contingency judgment have been made in contrast with so-called rule-based or statistical models (Allan \& Jenkins, 1983; Cheng \& Novick, 1992; Dickinson et al., 1984; Melz, Cheng, Holyoak, \& Waldmann, 1993; Shanks, 1993). At the heart of rule-based models is the normative assessment of binary contingencies as calculated by the $\Delta \mathrm{P}$ coefficient (Jenkins \& Ward, 1965). Contingencies among binary events can be represented in $2 \times 2$ tables as depicted at the bottom of Figures 1-2. The events symbolized on the left and at the top of such tables vary depending on the specific paradigm used (e.g., Pavlovian conditioning, operant conditioning, contingency judgment), but the overall structure remains constant. Here a CS, an action, or any other signal would correspond to $\mathrm{A}$, and the reinforcer or any outcome would correspond to $\mathrm{O}$. Taking the difference between the conditional probability of the outcome given the signal $(\mathrm{P}(\mathrm{O} \mid \mathrm{A})$ and the probability of the outcome in the absence of the signal $(\mathrm{P}(\mathrm{O} \mid \sim \mathrm{A})$ is a normative assessment of the contingency for that signal (A). To enable the comparison of associative models with the normative assessment of the contingency, the simulator provides the tables and calculates $\Delta \mathrm{P}$. Although it would be possible to calculate and plot $\Delta \mathrm{P}$ on a trial-by-trial basis, this calculation is often indeterminate, especially in the early trials when divisions by zero are encountered. Thus, strictly speaking, the comparison of an associative prediction with the normative one is best done at the end of a series. Nevertheless, when comparing associative models among themselves, one can use the simulator to study how the development of contingency judgments occurs trial by trial.

\section{FEATURES OF THE SIMULATOR}

The contingency tables are used to enter the frequencies of each type of trial in a series. Frequencies can be entered directly into the tables for A or B by pressing the Tab key until the desired table/cell gets the focus or by double clicking on a cell with the mouse. Typing in a new number overwrites the value on the screen, but a new model is computed only after all the desired individual frequencies are input and the Enter key is pressed. The simulator then updates the conditional probabilities, the $\Delta \mathrm{P}$ values, and the compound $(\mathrm{AB})$ table. If a value is indeterminate because of a division by zero, a question mark is displayed. The user can also modify the content of the contingency tables by entering new conditional probabilities directly or a new total number of trials. The frequencies in the table cells are adjusted proportionally.

In addition, the simulator validates the frequencies entered for $\mathrm{A}$ and $\mathrm{B}$ against the expected values for a single shared reinforcer. When the frequencies entered by the user imply a different total number of reinforcements for the two tables, the simulator warns the user and adjusts the frequencies for Table B to force a valid set given the values in Table A. If the solution provided by the simulator is not satisfactory, the user may enter new frequencies, making sure that the rule of a single shared reinforcer is respected and pressing the Enter key only after all the necessary cells have been modified. For experienced users, the warning messages displayed by the validation process can be disabled but the validation itself is always enforced. To disable the warnings, the user can click the appropriate check box in the lower left corner of the screen.

Other check boxes control various functions and display properties. The A, B, AB, and X (context) display boxes control whether to graph the strength of these stimuli. However, even if they are not graphed, their contributions are still computed internally.

The Alternate $\mathrm{X}$ checkbox allows for an alternative definition of the context. When applied to conditioning, the associative models define the complex of the conditioning apparatus as the context. This context is a continuous stimulus present on all trials. Alternatively, in the domain of contingency judgment, the context can often be defined as a discrete stimulus. For instance, in Baker, Mercier, ValléTourangeau, and Frank (1993), the CS is the camouflage while the context is the natural color of the tank. The tank can be camouflaged or not camouflaged, but not both at the same time. In this case, the context should gain associative strength only on the trials when it is present, which is to say, only when the CS is absent.

The Connect zero check box determines whether the simulator will plot curves connected to the zero point, or any other initial value, or simply plot from the first computed value onward.

When Autograph is on, the simulator automatically refreshes the plot after any parametric change. When Autoclear is on, any new plot clears the previous one; otherwise, plots are overlaid.

When the user is overlaying graphs to compare series of trials of different lengths, it is useful to first fix the scale of the $x$-axis to the length of the longer series via the Options - Xscaling subitem on the menu bar. Otherwise, the $x$-axis is automatically scaled to the number of trials of the most recent series. Another property of the $x$-axis, the number of trials to be included in each point plotted, is controlled by the Options - Block size menu. Once the model is graphed, it can be annotated with text by clicking in the plot area and typing text at the click position.

Which model to simulate is chosen with Options . Model, and it is possible to specify an initial associative strength other than zero before the first trial is computed with Options - Initial value. The parameters of the models are set to the desired values by clicking on the appropriate 
button (or tabbing to them, or pressing the left Alt key in combination with the underscored letter on the button label).

Two of the most powerful features of the simulator are provided by the Randomize and the Batch buttons. For any given contingency, associative models sometimes predict widely different final strengths depending on the order in which the trials have occurred. Blocking is a case in point. To quickly see the range of possible terminal values as well as the path to get there, the user can set up a contingency in the tables and then randomize the order of the trials and replot the model. To get a feel for the behavior of the model over a large set of different random orders, the user can do a batch of randomizations. In batch mode, the average curve for Stimulus $\mathrm{A}$ is plotted in addition (Autoclear off) or instead of (Autoclear on) the individual trials. Finally, if the exact values for associative strength are required, these are stored in a straightforward text file named "save.txt," which can be looked at with any text editor or word processor. Use the context-sensitive help (see below) to find out what the columns of numbers represent.

Another crucial file for the simulator is "simul.ini." It can be opened or saved via the File menu, and it contains a set of default values for all the parameters as well as the vectors defining each trial. The Open and Save As items in the File menu also allow the user to create and retrieve other files for individual projects.

The vectors defining each trial are displayed in the grid in the lower right corner of the screen. To specify individual trials, the user can give the focus to the grid by tabbing to it or single clicking the desired cell with the mouse. The active cell becomes surrounded by a dotted line. The user can change values by pressing 1 or 0 . It is possible to move around the grid using the arrow keys, PageUp, PageDown, CtrlHome, and CtrlEnd. The mouse can also operate the vertical scrollbar that appears on the right side of the grid, when there are more rows than can be displayed.

File - Print (or Ctrl P) is used to print a plot. To save the image of the simulation, the user presses the Prt Sc (Print Screen) key. This places a bitmap copy of the display in the Windows clipboard. The user can then paste it in other Windows applications such as word processors or graphics editors.

Finally, to obtain help on how to operate the simulator or details of the algorithms used for the core of the models, the user should give the focus to the desired button, menu item, or screen area and press the Fl key. Contextsensitive help will appear in its own window.

\section{Availability}

The simulator requires Windows 3.1 and 2MB RAM. It is available from the author by mail (send a formatted blank 1.44M disk) or via COMPsych. You can get RW.ZIP from the COMPsych BRMIC archive facility by anonymous FTP to Gluon. Hawk.Plattsburgh.Edu, path $=\mathrm{pub} /$ compsych/brmic.

\section{REFERENCES}

AL.LAN, L. G., \& JENKINS, H. M. (1983). The effect of representations of binary variables on judgment of influence. Learning \& Motivation, 14, 381-405.

Baker, A. G., Mercier, P., Vallée-Tourangeau, F., \& Frank, R. (1993). Selective associations and causality judgments: Presence of a strong causal factor may reduce judgments of a weaker one. Journal of Experimental Psychology. Learning, Memory, \& Cognition, 19, 414-432.

Bond, N. W., \& Siddle, D. A. T. (1987). Learning and motivation: Computer simulations of the Rescorla Wagner, Solomon-Corbit, and Schull models. Behavior Research Methods, Instruments, \& Computers, 19, 404-406.

ChapMAN, G. B. (1991). Tral order affects cue interaction in contingency judgment Journal of Experimental Psychology Learning, Memory, \& Cognition, 17, 837-854.

Chapman, G. B., \& Robbins, S. J. (1990). Cue interaction in human contingency judgment. Memory \& Cognition, 18, 537-545.

Cheng, P. W., \& Novick, L. R. (1992). Covariation in natural causal induction. Psychological Review, 99, 365-382.

Dickinson, A., Hall, G., \& Mackintosh, N. J. (1976). Surprise and the attenuation of blocking. Journal of Experimental Psychology: Animal Behavior Processes, 2, 313-322.

Dickinson, A., SHANKs, D.R., \& EVEnden, J. (1984). Judgement of actoutcome contingency The role of selective attribution. Quarterly Journal of Experimental Psychology, 36A, 29-50.

GluCK, M. A., \& Bower, G. H. (1988). From conditioning to category learning: An adaptive network model. Journal of Experimental Psychology General, 117, 227-247.

HaLL, G., \& PeARCE, J. M. (1982). Restoring the associability of a preexposed CS by a surprising event. Quarterly Journal of Experimental Psychology, 34, 127-140.

JENKINS, H. M., \& WARD, W. C. (1965). Judgment of contingencies between responses and outcomes. Psychological Monographs. General \& Applied, 79, 1-17

KAMIN, L. J. (1969). Predıctabıluty, surprise, attention, and conditioning. In B. A. Campbell \& R. M. Church (Eds.), Punishment and aversive behavior (pp. 279-298). New York: Appleton-Century-Crofts.

Lachnit, H., Schneider, R. L., LiPP, O. V., \& Kimmel, H. D. (1988), RWMODEL. A program in Turbo Pascal for simulating predictions based on the Rescorla-Wagner model of classical conditioning. Behavior Research Methods, Instruments, \& Computers, 20, 413-415.

LuBow, R. E., \& MOORE, A. V. (1959). Latent inhibition: The effect of nonreinforced pre-exposure to the conditional stimulus. Journal of Comparative \& Physiological Psychology, 52, 415-419.

MaCkINTOSH, N. J. (1971). An analysis of overshadowing and blocking. Quarterly Journal of Experimental Psychology, 23, 118-125

MaCkintosh, N. J. (1973). Stimulus selection: Learning to ignore stımuli that predict no change in reinforcement. In R. A. Hinde \& J. Stevenson-Hinde (Eds.), Constraints on learning (pp. 75-96). New York: Academic Press.

MACKINTOSH, N. J. (1975). Blocking of conditioned suppression. Role of the compound trial. Animal Behavior Processes, 1, 335-345.

Mackintosh, N. J. (1978), Cognitive or associative theories of conditioning: Implications of an analysis of blocking. In S. H Hulse, H. Fowler, \& W. K. Honig (Eds.), Cognitive processes in animal behavior (pp. 155-176). Hillsdale, NJ: Erlbaum.

Mackintosh, N J., Bygrave, D. J., \& Picton, B. M. B. (1977). Locus of the effect of a surprising reinforcer in the attenuation of blocking. Quarterly Journal of Experimental Psychology, 29, 327-336.

MACKINTOSH, N. J., \& TURNER, A. C. (1971). Blocking as a function of novelty of CS and predictability of UCS. Quarterly Journal of Experimental Psychology, 23, 359-366.

Melz, E. R., Cheng, P. W., Holyoak, K. J., \& Waldmann, M. R. (1993). Cue competition in human categorization: Contingency or the Rescorla- Wagner learning rule? Comments on Shanks (1991). Journal of Experimental Psychology Learning, Memory, \& Cognition, 19 , 1398-1410.

MERCIER, P., \& BAKER, A. G. (1985). Latent inhibition, habituation, and 
sensory preconditioning: A test of priming in short-term memory. Journal of Experimental Psychology: Animal Behavior Processes, 11, 485-501.

Pearce, J. M., \& Hall, G. (1980). A model for Pavlovian learning: Variations in the effectiveness of conditioned but not of unconditioned stimul. Psychological Review, 87, 532-552.

Pearce, J. M., Nicholas, D. J., \& Dickinson, A. (1982). Loss of associability by a conditioned inhibitor. Quarterly Journal of Experimental Psychology, 34, 149-162.

RESCORLA, R. A., \& WAGNER, A. R. (1972). A theory of Pavlovian conditioning: Variations in the effectiveness of reinforcement and nonreinforcement. In A. H. Black \& W. F. Prokasy (Eds.), Classical conditioning II Current theory and research (pp. 64-99). New York: Appleton-Century-Crofts.

SHANKS, D. R. (1985a). Contınuous monitoring of human contingency judgment across trials. Memory \& Cognition, 13, 158-167.

SHANKS, D. R. (1985b). Forward and backward blocking in human contingency judgement. Quarterly Journal of Experimental Psychology, 37B, 1-21.

SHANKs, D. R. (1986). Selective attribution and the judgment of causality. Learning \& Motivation, 17, 311-334.
SHANKS, D. R. (1989). Selectional processes in causality judgment. Memory \& Cognition, 17, 27-34.

SHANKS, D. R. (1991). Categorization by a connectionist network. Journal of Experimental Psychology. Learning, Memory, \& Cognition, 17, 433-443

ShanKs, D. R. (1993). Associative versus contingency accounts of category learning: Reply to Melz, Cheng, Holyoak, and Waldmann (1993). Journal of Experimental Psychology: Learning, Memory, \& Cognition, 19, 1411-1423.

Solomon, R. L., \& CoRBit, J. D. (1974). An opponent-process theory of motivation: 1. Temporal dynamics of affect. Psychological Review, 81, 119-145.

TuRner, C., \& Mackintosh, N. J. (1972). Stimulus selection and irrelevant stimuli in discrimination learning by pigeons. Journal of Comparative \& Physiological Psychology, 78, 1-9.

(Manuscript received November 4, 1994; revision accepted for publication April 4, 1995.) 